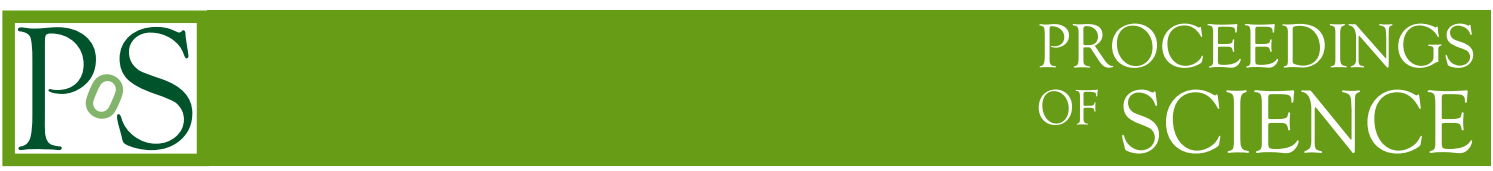

\title{
The ENUBET experiment
}
F. Terranova ${ }^{v, *}$ and F. Acerbi ${ }^{\mathrm{o}}$, G. Ballerini ${ }^{\mathrm{a}, \mathrm{b}}$, M. Bonesini $^{\mathrm{b}}$, A. Branca $^{\mathrm{j}, \mathrm{k}}$, C. Brizzolari ${ }^{\mathrm{b}, \mathrm{h}}$, G. Brunettij ${ }^{\mathrm{j}, \mathrm{k}}$, M. Calviani ${ }^{\mathrm{m}}$, S. Capelli ${ }^{\mathrm{a}, \mathrm{b}}$, S. Carturan ${ }^{\mathrm{u}}$, M.G. Catanesi ${ }^{1}$, S. Cecchini ${ }^{\mathrm{c}}$, N. Charitonidis ${ }^{\mathrm{m}}$, F. Cindolo $^{\mathrm{c}}$, G. Collazuol $^{\mathrm{k}, \mathrm{j}}$, E. Conti $^{\mathrm{j}}$, F. Dal Corso ${ }^{j}$, C. Delogu ${ }^{\mathrm{j}, \mathrm{k}}$, G. De Rosa ${ }^{\mathrm{p}, \mathrm{q}}$, A. Falcone $^{\mathrm{b}, \mathrm{h}}$, B. Goddard ${ }^{\mathrm{m}}$, A. Gola ${ }^{\mathrm{o}}$, C. Jollet $^{\mathrm{d}, \mathrm{s}}$, V. Kein ${ }^{\mathrm{m}}$, B. Klicek ${ }^{\mathrm{t}}$, Y. Kudenko ${ }^{\mathrm{r}}$, M. Laveder ${ }^{\mathrm{j}, \mathrm{k}}$, A. Longhin ${ }^{\mathrm{j}, \mathrm{k}}$, L. Ludovici ${ }^{\mathrm{f}}$, E. Lutsenko ${ }^{\mathrm{a}, \mathrm{b}}$, L. Magaletti ${ }^{1}$, G. Mandrioli ${ }^{\mathrm{c}}$, A. Margotti $^{\mathrm{c}}$, V. Mascagna ${ }^{\mathrm{a}, \mathrm{b}}$, N. Mauri ${ }^{\mathrm{C}}$, L. Meazza ${ }^{\text {bh }}$, A. Meregaglias ${ }^{\mathrm{s}}$, M. Mezzetto $^{\mathrm{j}}$, M. Nessi $^{\mathrm{m}}$, A. Paoloni $^{\mathrm{e}}$, M. Pari ${ }^{k, j}$, E. Parozzi ${ }^{\text {b,m }}$, L. Pasqualini ${ }^{\mathrm{c}, \mathrm{g}}$, G. Paternoster ${ }^{\mathrm{o}}$, L. Patrizii ${ }^{\mathrm{c}}$, M. Pozzato ${ }^{\mathrm{c}}$, M. Prest ${ }^{\mathrm{a}, \mathrm{b}}$, F. Pupilli ${ }^{\mathrm{e}}$, E. Radicioni ${ }^{1}$, C. Riccio ${ }^{\mathrm{p}, \mathrm{q}}$, A. C. Ruggeri ${ }^{\mathrm{p}}$, C. Scian ${ }^{\mathrm{k}}$, G. Sirri $^{\mathrm{c}}$,

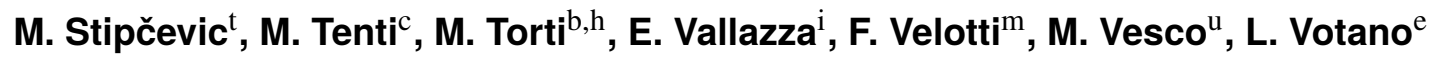

a Phys. Dep. Università degli Studi dell'Insubria, Como, Italy.

b INFN, Sezione di Milano-Bicocca, Milano, Italy.

${ }^{\mathrm{c}}$ INFN, Sezione di Bologna, Bologna, Italy.

d IPHC, Université de Strasbourg, CNRS/IN2P3, Strasbourg, France.

e INFN, Laboratori Nazionali di Frascati, Frascati (Rome), Italy.

f INFN, Sezione di Roma 1, Rome, Italy.

g Phys. Dep. Università di Bologna, Bologna, Italy.

${ }^{\mathrm{h}}$ Phys. Dep. Università di Milano-Bicocca, Milano, Italy.

${ }^{i}$ INFN Sezione di Trieste, Trieste, Italy.

j INFN Sezione di Padova, Padova, Italy.

k Phys. Dep. Università di Padova, Padova, Italy.

${ }^{1}$ INFN Sezione di Bari, Bari, Italy.

${ }^{m}$ CERN, Geneva, Switzerland.

${ }^{\mathrm{n}}$ Phys. Dep. Università La Sapienza, Rome, Italy.

${ }^{\circ}$ Fondazione Bruno Kessler (FBK) and INFN TIFPA, Trento, Italy.

p INFN, Sezione di Napoli, Napoli, Italy.

q Phys. Dep. Università degli Studi di Napoli Federico II, Napoli, Italy.

${ }^{\mathrm{r}}$ Institute of Nuclear Research of the Russian Academy of Science, Moscow, Russia.

${ }^{s}$ CENBG, Université de Bordeaux, CNRS/IN2P3, Gradignan, France.

${ }^{\mathrm{t}}$ Center of Excellence for Advanced Materials and Sensing Devices, Ruder Boskovic Institute, Zagreb, Kroatia. 
u INFN Laboratori Nazionali di Legnaro, Legnaro (PD), Italy.

${ }^{v}$ Univ. of Milano-Bicocca and INFN, Sez. di Milano-Bicocca, Piazza della Scienza 3, Milano, Italy. E-mail: francesco.terranova@cern.ch

The CERN NP06/ENUBET experiment is designing the first "monitored neutrino beam", i.e. a neutrino beam with unprecedented control of the flux, energy and flavor of the neutrinos at source. The original aim of ENUBET was to monitor the $v_{e}$ production mostly by the detection of large-angle positrons from three-body semileptonic decays of kaons: $K^{+} \rightarrow e^{+} \pi^{0} v_{e}$. Over the years, the ENUBET technique has been extended to cover also the monitoring of the $v_{\mu}$ from kaon and pion decays. In this paper, we present these new developments and the overall status of the project.

40th International Conference on High Energy physics - ICHEP2020

July 28 - August 6, 2020

Prague, Czech Republic (virtual meeting)

${ }^{*}$ Speaker 


\section{ENUBET and the monitored neutrino beams}

ENUBET is aimed at designing a narrow-band neutrino beam at the $\mathrm{GeV}$ scale, measuring the flux and flavor at $1 \%$ level, and the energy of the neutrinos produced at source with a precision of $\sim 10 \%$. It is the first "monitored neutrino beam" [1] and the core technology for a new generation of short-baseline experiments to achieve a $1 \%$ precision on the $v_{e}$ and $v_{\mu}$ cross-sections and remove all biases due the $v_{\mu}$ energy reconstruction. It is essential to lower to $<3 \%$ the systematic budget of DUNE and HyperKamiokande and enhance remarkably their discovery reach (equivalent to doubling the DUNE mass). Finally, this facility is the most natural follow-up of the previous generation of cross-section experiments - including the possibility to upgrade the CERN ProtoDUNE or the Fermilab SBN physics programme for a new experimental campaign focused on cross-section measurements and physics beyond the Standard Model (see e.g. [2, 3]).

\section{Beamline}

The beamline of ENUBET produces a narrow-band beam of neutrinos with a typical energy spread of $10 \%$ and a large angle between the neutrinos and the target axis $\left(14.8^{\circ}\right)$. This displacement ensures that the $\mathrm{GeV}$ neutrinos produced in the first non-instrumented part of the beamline (decay of neutral hadrons, early decay-in-flight of charged particles) do not reach the neutrino detector. In the latest version of the ENUBET beamline, this is achieved by two dipoles and a set of quadrupoles shown in Fig. 1. An additional advantage of this configuration is the large distance between the proton dump, i.e. the element stopping the primary protons that have not interacted with the target, and the neutrino detector. Again, this reduces the amount of non-monitored neutrinos that reach the detector in the energy range of DUNE or Hyper-Kamiokande. Possible low-energy residual neutrinos from the decay-at-rest can be identified by the detector and used for ancillary physics measurements [4].

At the time of writing, the new beamline has been fully simulated with G4Beamline and FLUKA, and the optimization of the target and collimators is in progress. This beamline is based on a purely static focusing system that dilutes the protons up to $4 \mathrm{~s}$. In such a way, the instantaneous rate in the instrumented decay tunnel does not exceed a few hundreds of $\mathrm{kHz}$ and the pile-up contribution to the background is marginal. The corresponding fluxes are comparable to the previous version and a full evaluation is in progress. The same beamline is being re-optimized to account for a horn-based system pulsed at 2-10 ms during the flat top of the proton accelerator. The proton extraction scheme, proposed for the first time in [5], has been demonstrated in 2018 at the CERN-SPSC at the $20 \mathrm{~ms}$ level and the latest tuning based on the simulations of the SPSC lattice achieved $<10 \mathrm{~ms}$ and will be tested at the end of the CERN Long-Shutdown 2 (LS2) [6]. This proton extraction scheme can be used in the static focusing beamline, as well, to provide a time structure that is employed by the detector for cosmic ray suppression.

\section{Tunnel instrumentation}

The core of the monitored beams are a rich instrumentation of the decay tunnel that provides the rate of the leptons produced at large angles. The ENUBET tunnel instrumentation is based on 


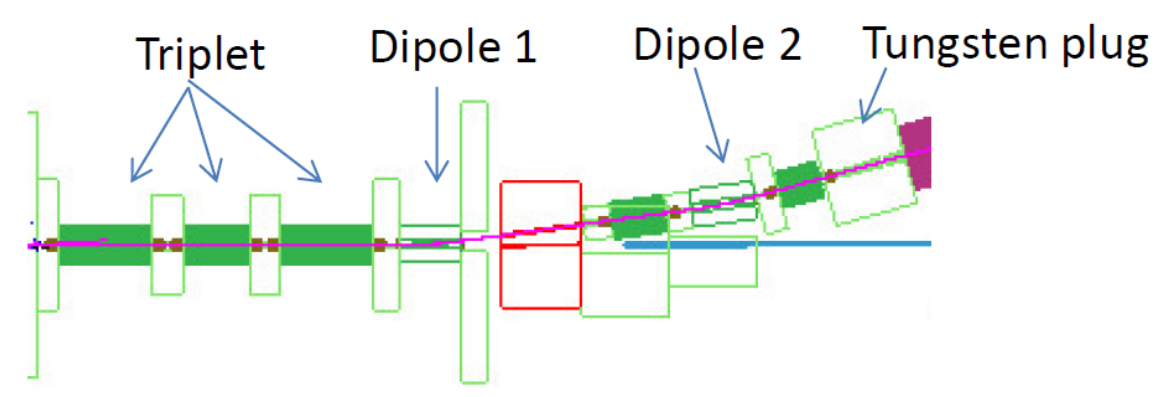

Figure 1: Bending section of the ENUBET beamline. The section is located between the target area and the decay tunnel.

a calorimeter for $e^{+} / \pi^{+}$separation and of an inner light-weight photon veto for $e^{+} / \pi^{0}$ separation. This detector also provides the absolute timing of the events and is thus called the " $t_{0}$-layer". We carried out the detector R\&D between 2016 and 2018, mostly at the CERN East Experimental Area [7]. The final choice is an iron-scintillator sampling calorimeter divided into modules that sample the showers every 4.3 radiation lengths. The scintillator light is collected by WLS fibers (Y11 by Kuraray) running along the lateral edge of the tiles and bundled on top of the detector. The photosensors that will be employed are SiPMs produced by Fondazione Bruno Kessler (FBK). The photon veto is based on plastic scintillators, whose light is transported by WLS fibers toward the upper part, where the SiPMs are located. A detailed description of the performance of the prototype is given in [8]. The final ENUBET Demonstrator is a $3 \mathrm{~m}$ long instrumented decay tunnel with a radius of $1 \mathrm{~m}$ that will be built in 2021 and tested at CERN after LS2.

\section{Particle reconstruction in the decay tunnel}

Since 2017, we have developed a dedicated event builder to identify and reconstruct positrons in the calorimeter with high efficiency and purity. More recently, the event builder for the reconstruction of muons has been finalized, too. In the muon event builder, the event reconstruction starts with the identification of a seed. The seed for the muon reconstruction is a visible energy deposit in the calorimeter cells (Lateral readout Compact Modules, LCMs) of the innermost layer compatible with a mip i.e. with an energy between 5 and $15 \mathrm{MeV}$. Around the seed, all LCMs and t0-layer deposits compatible in space with a muon track are clustered together and constitute the candidate muon event. The search of the energy deposits is performed taking into account the expected trajectory of the muon, a straight line from the inner radial layer of LCMs toward the outer layers in the forward direction. The clustering exploits the time of the energy deposit in the LCMs. By 2020, the energy deposit is simulated in the most realistic manner plugging the entire waveform recorded by the digitizers that read the SiPM signal without amplification. The algorithms for peak identification and pile-up subtraction are under development but preliminary results show that a timing precision of $\sim 1 \mathrm{~ns}$ can be achieved for each LCM even in the presence of pile-up or afterpulses. Correlated noise may impact on the energy resolution of positrons $(\simeq 17 \% / \sqrt{(} E[\mathrm{GeV}])[8]$ and low cross-talk devices developed by FBK [9] are being considered for the final detector. 


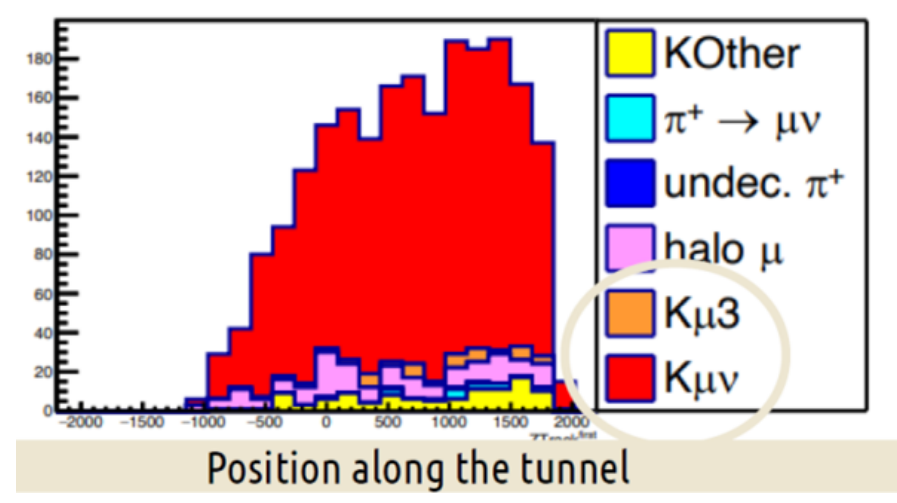

Figure 2: Distribution of selected muons (signal and background) as a function of the position in the $40 \mathrm{~m}$ instrumented decay tunnel. The signal is highlighted by the beige circle.

We identified a set of variables that give information on the reconstructed event topology both for positrons and muons. These variables are used by a Neural Network (NN) based on the Root TMVA package and the event is selected cutting on the output classifier of the network, which ranges from 0 (background) to 1 (pure $e^{+}$or $\mu^{+}$candidate). The preliminary performance obtained for muons from $\mathrm{K}^{+}$shows a selection efficiency of about $33 \%$ dominated by the geometrical efficiency, and a $\mathrm{S} / \mathrm{N}$ of $\sim 6$. Fig. 2 reports the distribution of the impact point along the tunnel for the reconstructed muons after the cut on the NN classifier. The corresponding efficiency and S/N for positrons are $24 \%$ and 2.1 , respectively.

\section{5. $v_{\mu}$ monitoring from pion decays}

The original ENUBET technique is not suited to monitor muons from the $\pi^{+} \rightarrow \mu^{+} v_{\mu}$ decay since the muons are produced in the forward direction and impinge into the hadron dump without crossing the instrumented walls of the tunnel. Thanks to the slow extraction, however, the rate of these muons goes from 1 to $10 \mathrm{MHz} / \mathrm{cm}^{2}$ and the muon range (i.e. the muon energy) can be measured instrumenting the dump with fast and radiation hard detectors (silicon pads, polysiloxane scintillator cells [10], etc.) at different depths. The instrumented dump can then monitor the production rate with systematics much better than the standard $15 \%$ precision that can be obtained in high energy wide-band beams by ionization chambers and identify energy, position and rate at the single-particle level. NP06/ENUBET is therefore potentially equipped with a tool to determine the flux of the leading $v_{\mu}$ with a precision similar to the flux of $v_{e}$, enhancing remarkably the physics reach of the facility.

\section{Systematic evaluation and physics performance}

Since late 2019, we have started the full systematic assessment to demonstrate that ENUBET can achieve the $1 \%$ goal on the $v_{e}$ and $v_{\mu}$ fluxes. The technique we chose is inspired by T2K and uses as priors all the information from the simulation like in a standard beam, including hadroproduction data. Unlike $\mathrm{T} 2 \mathrm{~K}$, we include in the priors also the monitoring information: the rate, energy, and angular distribution of positrons and muons in the decay tunnel, and the information 
from the instrumented hadron dump. These additional priors constrain the flux once we consider systematic variations on the beam parameters, secondary production yields, the uncertainty in the transport and interactions of the secondaries in the transfer line and the instrumentation efficiency. We plan to release the complete systematic matrix by the end of 2021.

ENUBET is thus able to provide a sample of about $10^{4} v_{e} \mathrm{CC}$ and $10^{6} v_{\mu} \mathrm{CC}$ in a $500 \mathrm{t}$ detector located $50 \mathrm{~m}$ after the hadron dump (baseline: $90 \mathrm{~m}$ ) monitored with an expected precision of $1 \%$. Using the "off-axis narrow-band technique" described in [2], we can provide a measurement of the neutrino energy just by locating the position of the interaction vertex in the detector, i.e. without relying on final state particle reconstruction. If we define $R$ as the distance of the interaction vertex from the beam axis, the relative beam energy width $\left(\Delta E_{v} / E_{v}\right)$ at fixed $R$ (i.e. the neutrino energy resolution for the $v_{\mu}$ from pion decay) is $8 \%$ for $R \simeq 50 \mathrm{~cm}$ with $\left\langle E_{v}\right\rangle \sim 3 \mathrm{GeV}$ and $22 \%$ for $R \simeq 250 \mathrm{~cm}$ with $\left\langle E_{v}\right\rangle \simeq 0.7 \mathrm{GeV}$.

\section{Acknowledgments}

We are grateful to the organizers of ICHEP2020 for the excellent discussion framework despite the limitations due to the COVID19 outbreak. This project has received funding from the European Research Council (ERC) under the European Union's Horizon 2020 Research and Innovation programme (Grant Agreement no. 681647) and by the Italian Ministry of Education and Research - MIUR (Bando "FARE", progetto NuTech). It is also supported by the Dep. of Physics "G. Occhialini”, Univ. of Milano-Bicocca (project 2018-CONT-0128).

\section{References}

[1] A. Longhin, L. Ludovici and F. Terranova, Eur. Phys. J. C 75 (2015) 155.

[2] F. Acerbi et al., CERN-SPSC-2018-034, SPSC-I-248, Geneva, 2018.

[3] L. Delgadillo and P. Huber, "Sterile neutrino searches at tagged kaon beams," arXiv:2010.10268 [hep-ph].

[4] A. A. Aguilar-Arevalo et al. [MiniBooNE], Phys. Rev. Lett. 120 (2018) 141802.

[5] A. Berra et al., CERN-SPSC-2016-036, SPSC-EOI-014, Geneva, 2016.

[6] M. Pari, M. Fraser, B. Goddard, V. Kain, L. Stoel and F. Velotti, Proceedings of IPAC 2019, doi:10.18429/JACoW-IPAC2019-WEPMP035.

[7] A. Berra et al., Nucl. Instrum. Meth. A 830 (2016) 345; A. Berra et al., IEEE Trans. Nucl. Sci. 64 (2017) 1056; G. Ballerini et al., JINST 13 (2018) P01028. F. Acerbi et al., JINST 14 (2019) P02029.

[8] F. Acerbi, et al. JINST 15 (2020) P08001.

[9] A. Gola et al. Sensors 19 (2019) 308.

[10] F. Acerbi et al. Nucl. Instrum. Meth. A 956 (2020) 163379. 